\title{
Palladium-Catalyzed Synthesis of 2,1'-Disubstituted Tetrahydrofurans from $\gamma$-Hydroxy Internal Alkenes. Evidence for Alkene Insertion into a Pd-O Bond and Stereochemical Scrambling via $\beta$-Hydride Elimination
}

\author{
Michael B. Hay and John P. Wolfe* \\ Department of Chemistry, University of Michigan, 930 N. University Ave., Ann Arbor, MI, \\ 48109-1055
}

\section{Supporting Information}

Experimental procedures for synthesis of substrates, characterization data for all new compounds, descriptions of deuterium labeling experiments, and descriptions of stereochemical assignments (21 pages).

\section{Table of Contents}

General Considerations

Preparation and Characterization of Alcohol Substrates

Characterization Data for Tetrahydrofuran Products

Assignment of Stereochemistry

Deuterium Labeling Studies

References
S-1

S-2

S-5

S-12

S-16

S-21

\section{General Considerations}

All reactions were carried out under an argon or nitrogen atmosphere in oven- or flame-dried glassware. All catalysts and reagents were obtained from commercial sources and were used without 
further purification. Compounds $\mathbf{1 , 2}, \mathbf{3}, \mathbf{4 a}$, and 10a have been reported previously. ${ }^{1}$ Toluene, THF, diethyl ether, and methylene chloride were purified using a GlassContour solvent purification system. Yields refer to isolated yields of compounds estimated to be $\geq 95 \%$ pure as determined by ${ }^{1} \mathrm{H}$ NMR and either capillary GC (known compounds) or combustion analysis (new compounds). The yields reported in the supporting information describe the result of a single experiment, whereas the yields reported in Tables 1-2 are average yields of two or more experiments. Thus, the yields reported in the supporting information may differ from those shown in Tables 1-2.

\section{Preparation and Characterization of Alcohol Substrates}

General Procedure 1: Addition of MeMgBr to Esters. An oven or flame dried flask was purged with argon or nitrogen and charged with $\mathrm{MeMgBr}$ (3 equiv, 3.0 $\mathrm{M}$ in diethyl ether). Additional ether was added to provide a $1.0 \mathrm{M}$ solution, which was then cooled to $0^{\circ} \mathrm{C}$. The appropriate ester was added dropwise via syringe and the resulting mixture was warmed to $\mathrm{rt}$ and stirred for $2-4 \mathrm{~h}$ until the starting material was found to be completely consumed as judged by TLC analysis. A solution of saturated aqueous $\mathrm{NH}_{4} \mathrm{Cl}$ (1:1 by volume with reaction mixture) was added dropwise and the resulting mixture was then diluted with ethyl acetate (1:1 by volume with reaction mixture). The layers were separated and the aqueous layer was extracted with ethyl acetate. The combined organic layers were dried over anhydrous $\mathrm{Na}_{2} \mathrm{SO}_{4}$, filtered, and concentrated in vacuo. The crude tertiary alcohol product was then purified by flash chromatography on silica gel.

General Procedure 2: Johnson Orthoester Claisen Rearrangements. ${ }^{2}$ A round bottom flask equipped with a short path distillation head and a recovery flask was charged with the appropriate allylic alcohol, triethyl orthoacetate (10 equiv), and pivalic acid ( 0.06 equiv). The mixture was heated to $100{ }^{\circ} \mathrm{C}$ with stirring for $2 \mathrm{~h}$ and ethanol was continuously removed by distillation. The mixture was 
then heated to $140{ }^{\circ} \mathrm{C}$ for $12 \mathrm{~h}$ until the starting material had been completely consumed as judged by GC analysis. The reaction mixture was cooled to room temperature and diluted with ethyl acetate (1:1 by volume). A solution of $1 \mathrm{M}$ aqueous $\mathrm{HCl}$ (1:1 by volume) was slowly added and the resulting biphasic mixture was stirred for $1 \mathrm{~h}$ at $\mathrm{rt}$. The layers were separated and the organic layer was washed with water $(2 \times 50 \mathrm{~mL})$, and saturated sodium bicarbonate $(1 \times 50 \mathrm{~mL})$. The organic layer was then dried over anhydrous $\mathrm{Na}_{2} \mathrm{SO}_{4}$, filtered, and concentrated in vacuo. The crude ester product was used without further purification.

(Z)-2-Methylhept-5-en-2-ol (9). ${ }^{3}$ (Z)-Hex-4-enoic acid ethyl ester ${ }^{4}(1.92 \mathrm{~g}, 13.5 \mathrm{mmol})$ was treated with $\mathrm{MeMgBr}(18.6 \mathrm{~mL}, 56 \mathrm{mmol}, 3.0 \mathrm{M}$ in diethyl ether) according to general procedure 1. The crude material was purified by fractional distillation $\left(140{ }^{\circ} \mathrm{C}, 760 \mathrm{~mm} \mathrm{Hg}\right)$ to afford $1.36 \mathrm{~g}(78 \%)$ of the title compound as a colorless oil. ${ }^{1} \mathrm{H}$ NMR $\left(500 \mathrm{MHz}, \mathrm{CDCl}_{3}\right) \delta 5.50-5.36(\mathrm{~m}, 2 \mathrm{H}), 2.16-2.10$ $(\mathrm{m}, 2 \mathrm{H}), 1.62(\mathrm{~d}, J=6.0 \mathrm{~Hz}, 3 \mathrm{H}), 1.55-1.51(\mathrm{~m}, 2 \mathrm{H}), 1.29(\mathrm{~s}, 1 \mathrm{H}), 1.23(\mathrm{~s}, 6 \mathrm{H})$.

( \pm )-1-Cyclohex-2-enyl-2-methylpropan-2-ol (16). Cyclohex-2-enylacetic acid ethyl ester ${ }^{5}$ was treated with $\mathrm{MeMgBr}(14.8 \mathrm{~mL}, 44.4 \mathrm{mmol}, 3.0 \mathrm{M}$ in diethyl ether) according to general procedure 1 to afford $1.32 \mathrm{~g}(58 \%)$ of the title compound as a colorless oil. ${ }^{1} \mathrm{H}$ NMR $\left(400 \mathrm{MHz}, \mathrm{CDCl}_{3}\right) \delta 5.51(\mathrm{~m}$, $2 \mathrm{H}), 2.16-2.12(\mathrm{~m}, 1 \mathrm{H}), 1.84-1.80(\mathrm{~m}, 2 \mathrm{H}), 1.74-1.68(\mathrm{~m}, 1 \mathrm{H}), 1.59-1.53(\mathrm{~m}, 1 \mathrm{H}), 1.45-1.30$ (m, 3 H), 1.22-1.13 (m, $1 \mathrm{H}), 1.11(\mathrm{~s}, 7 \mathrm{H}) ;{ }^{13} \mathrm{C} \mathrm{NMR}\left(100 \mathrm{MHz}, \mathrm{CDCl}_{3}\right) \delta$ 132.9, 126.5, 71.4, 50.0, 31.4, 30.9, 29.9, 29.8, 24.9, 21.2; IR (film) 3368, $1147 \mathrm{~cm}^{-1}$. Anal calcd for $\mathrm{C}_{10} \mathrm{H}_{18} \mathrm{O}: \mathrm{C}, 77.87 ; \mathrm{H}, 11.76$. Found: C, 77.57; H, 11.76 . 
4-Cyclohex-1-enyl-2-methylbutan-2-ol (17) A flame dried flask was cooled under a stream of argon and charged with diisopropyl amine $(1.10 \mathrm{~g}, 10.9 \mathrm{mmol})$, DMPU (0.80 g, $6.24 \mathrm{mmol})$, and THF (3.1 mL). The mixture was cooled to $0{ }^{\circ} \mathrm{C}$ and a solution of $n$-butyllithium $(6.5 \mathrm{~mL}, 10.4 \mathrm{mmol}, 1.6 \mathrm{M}$ in hexanes) was added slowly. The reaction was stirred for $30 \mathrm{~min}$ at $0{ }^{\circ} \mathrm{C}$ and was then cooled to $-78^{\circ} \mathrm{C}$, whereupon neat tert-butyl acetate $(6.5 \mathrm{~mL}, 10.4 \mathrm{mmol})$ was added dropwise. The solution was stirred for $1 \mathrm{~h}$ at $-78^{\circ} \mathrm{C}$, then 1-bromomethylcyclohexene ${ }^{6}(2.0 \mathrm{~g}, 11.4 \mathrm{mmol})$ was added. The reaction mixture was stirred at $-78^{\circ} \mathrm{C}$ for $1 \mathrm{~h}$ and then warmed to rt with stirring over $2 \mathrm{~h}$. The reaction mixture was then quenched with saturated aqueous $\mathrm{NH}_{4} \mathrm{Cl}(10 \mathrm{~mL})$ and stirred for 10 minutes at rt. Ethyl acetate $(20 \mathrm{~mL})$ was added, the layers were separated, and the aqueous layer was extracted with ethyl acetate $(3 \times 10 \mathrm{~mL})$. The combined organic layers were dried over anhydrous $\mathrm{MgSO}_{4}$, filtered, and concentrated in vacuo to afford crude 3-cyclohex-1-enylpropionic acid tert-butyl ester. Treatment of the crude ester with $\mathrm{MeMgBr}(6.3 \mathrm{~mL}, 18.9 \mathrm{mmol}, 3.0 \mathrm{M}$ in diethyl ether) following general procedure 1 afforded $0.32 \mathrm{~g}$ (40\% over two steps) of 4-cyclohex-1-enyl-2-methylbutan-2-ol as a clear oil. ${ }^{1} \mathrm{H}$ NMR (400 MHz, $\left.\mathrm{CDCl}_{3}\right) \delta 5.44-5.40(\mathrm{~m}, 1 \mathrm{H}), 2.04-1.93(\mathrm{~m}, 6 \mathrm{H}), 1.64-1.54(\mathrm{~m}, 7 \mathrm{H}), 1.21(\mathrm{~s}, 6 \mathrm{H})$; ${ }^{13} \mathrm{C}$ NMR $\left(100 \mathrm{MHz}, \mathrm{CDCl}_{3}\right) \delta 138.0,120.9,120.8,77.2,41.5,32.7,29.1,28.3,25.2,22.9,22.4$; IR (film) $3368,1147 \mathrm{~cm}^{-1}$. Anal calcd for $\mathrm{C}_{11} \mathrm{H}_{20} \mathrm{O}: \mathrm{C}, 78.51 ; \mathrm{H}, 11.98$. Found: C, 78.30; H, 12.09 .

4-Cyclopent-1-enyl-2-methyl-butan-2-ol (18). 3-Iodopropionic acid ethyl ester (3.40 g, 15 mmol) was converted to the corresponding alkylzinc reagent and cross-coupled with cyclopent-1-enyl $\operatorname{triflate}^{7}(2.16 \mathrm{~g}, 10 \mathrm{mmol})$ using a procedure reported by Yoshida for the cross-coupling of alkylzinc reagents with cyclohexenyl triflates. ${ }^{8}$ This procedure afforded $670 \mathrm{mg}(40 \%)$ of 3-cyclopent-1enylpropionic acid ethyl ester. ${ }^{1} \mathrm{H}$ NMR $\left(500 \mathrm{MHz}, \mathrm{CDCl}_{3}\right) \delta 5.35-5.34(\mathrm{~m}, 1 \mathrm{H}), 4.13(\mathrm{q}, J=7.0 \mathrm{~Hz}$, 
$2 \mathrm{H}), 2.46(\mathrm{t}, J=7.0 \mathrm{~Hz}, 2 \mathrm{H}), 2.40-2.36(\mathrm{~m}, 2 \mathrm{H}), 2.31-2.27(\mathrm{~m}, 2 \mathrm{H}), 2.25-2.22(\mathrm{~m}, 2 \mathrm{H}), 1.85$ (p, $J$ $=7.0 \mathrm{H}, 2 \mathrm{H}), 1.25(\mathrm{t}, J=7.0 \mathrm{~Hz}, 3 \mathrm{H})$.

3-Cyclopent-1-enylpropionic acid ethyl ester (670 mg, $3.98 \mathrm{mmol}$ ) was treated with $\mathrm{MeMgBr}$ (4.0 mL, $12.0 \mathrm{mmol}, 3.0 \mathrm{M}$ in diethyl ether) according to general procedure 1 to afford $345 \mathrm{mg}$ (56\%) of the title compound as a colorless oil. ${ }^{1} \mathrm{H}$ NMR $\left(500 \mathrm{MHz}, \mathrm{CDCl}_{3}\right) \delta 5.36-5.33(\mathrm{~m}, 1 \mathrm{H}), 2.29-2.22$ (m, 4 H), 2.14-2.11 (m, $2 \mathrm{H}), 1.83(\mathrm{p}, J=8.0 \mathrm{~Hz}, 2 \mathrm{H}), 1.64-1.60(\mathrm{~m}, 2 \mathrm{H}), 1.47(\mathrm{~s}, 1 \mathrm{H}), 1.21(\mathrm{~s}, 6 \mathrm{H})$; ${ }^{13} \mathrm{C}$ NMR (125 MHz, $\left.\mathrm{CDCl}_{3}\right) \delta 144.8,123.1,70.9,41.5,35.1,32.3,29.1,25.9,23.3$; IR (film) 3368 $\mathrm{cm}^{-1}$. MS (EI) $\mathrm{m} / z$ 154.1360 (154.1360 calcd for $\left.\mathrm{C}_{10} \mathrm{H}_{18} \mathrm{O}\right)$.

\section{Characterization Data for Tetrahydrofuran Products}

( \pm )-(4R,5R)-4-Biphenyl-4-yl-5-ethyl-2,2-dimethyltetrahydrofuran (4b). This compound was isolated from the mixture of $\mathbf{4 a}, \mathbf{4 b}$, and $\mathbf{4} \mathbf{c}$ by preparative HPLC and was obtained as a $\sim 5: 1$ mixture of $\mathbf{4 b}$ and $\mathbf{4 c}$. Data are given for $\mathbf{4 b} .{ }^{1} \mathrm{H}$ NMR $\left(500 \mathrm{MHz}, \mathrm{CDCl}_{3}\right) \delta 7.59(\mathrm{~d}, J=8.0 \mathrm{~Hz}, 2 \mathrm{H}), 7.55(\mathrm{~d}, J$ $=8.0 \mathrm{~Hz}, 2 \mathrm{H}), 7.44(\mathrm{t}, J=8.0 \mathrm{~Hz}, 2 \mathrm{H}), 7.36-7.31(\mathrm{~m}, 3 \mathrm{H}), 3.93(\mathrm{ddd}, J=4.0,6.2,10.0 \mathrm{~Hz}, 1 \mathrm{H})$, $3.11(\mathrm{ddd}, J=8.0,9.7,11.5 \mathrm{~Hz}, 1 \mathrm{H}), 2.25(\mathrm{dd}, J=8.0,12.5 \mathrm{~Hz}, 1 \mathrm{H}), 2.05(\mathrm{t}, J=11.5 \mathrm{~Hz}, 1 \mathrm{H})$, 1.66-1.60 (m, $1 \mathrm{H}), 1.55-1.48(\mathrm{~m}, 1 \mathrm{H}), 1.47(\mathrm{~s}, 3 \mathrm{H}), 1.34(\mathrm{~s}, 3 \mathrm{H}), 0.92(\mathrm{t}, J=7.5 \mathrm{~Hz}, 3 \mathrm{H}) ;{ }^{13} \mathrm{C}$ NMR $\left(125 \mathrm{MHz}, \mathrm{CDCl}_{3}\right) \delta 140.8,140.1,139.4,128.7,128.1,127.2,127.1,126.9,85.9,79.5,50.5,48.6$, 29.6, 29.4, 26.7, 10.1. MS (EI) $m / z 280.1827\left(280.1827\right.$ calcd for $\left.\mathrm{C}_{20} \mathrm{H}_{24} \mathrm{O}\right)$.

The structure of isomer $4 \mathbf{c}$ was assigned based on comparison of the literature ${ }^{1} \mathrm{H}$ and ${ }^{13} \mathrm{C}$ NMR data for the closely related compound 2,2-dimethyl-5-phenethyltetrahydrofuran ${ }^{9}$ to the additional signals present in the ${ }^{1} \mathrm{H}$ and ${ }^{13} \mathrm{C}$ NMR spectra of the $4 \mathbf{b} / 4 \mathbf{c}$ mixture. 
(士)-(1'S,5R)-2,2-Dimethyl-5-(1'-o-tolylethyl)tetrahydrofuran (11a). Reaction of (E)-2methylhept-5-en-2-ol (64 mg, $0.5 \mathrm{mmol})$ with 2-bromotoluene (171 $\mathrm{mg}, 1.0 \mathrm{mmol})$ following the general procedure afforded $94 \mathrm{mg}(86 \%)$ of the title compound as a pale yellow oil. This material was obtained as a ca. 6:1 mixture of diastereomers as judged by ${ }^{1} \mathrm{H}$ NMR analysis (the crude reaction mixture contained a 3:1 mixture of diastereomers). Data are for the major diastereomer. ${ }^{1} \mathrm{H}$ NMR (400 $\left.\mathrm{MHz}, \mathrm{CDCl}_{3}\right) \delta 7.31-7.10(\mathrm{~m}, 4 \mathrm{H}), 4.17-4.11(\mathrm{~m}, 1 \mathrm{H}), 3.23(\mathrm{p}, J=6.4 \mathrm{~Hz}, 1 \mathrm{H}), 2.38(\mathrm{~s}, 3 \mathrm{H})$, 1.91-1.80 (m, $1 \mathrm{H}), 1.79-1.73(\mathrm{~m}, 1 \mathrm{H}), 1.72-1.65$ (m, $2 \mathrm{H}), 1.29-1.22$ (m, $9 \mathrm{H}) ;{ }^{13} \mathrm{C}$ NMR (100 MHz, $\left.\mathrm{CDCl}_{3}\right) \delta 142.6,135.9,130.0,126.4,125.8,125.7,81.8,80.2,38.8,38.6,28.7,28.4,28.0,19.8,15.9 ;$ IR (film) $1364 \mathrm{~cm}^{-1}$. Anal calcd for $\mathrm{C}_{15} \mathrm{H}_{22} \mathrm{O}: \mathrm{C}, 82.52 ; \mathrm{H}, 10.16$. Found C, 82.72; H, 9.86.

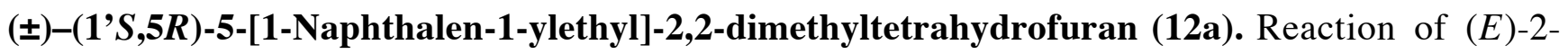
methylhept-5-en-2-ol (64 mg, $0.5 \mathrm{mmol})$ with 1-bromonaphthalene (207 $\mathrm{mg}, 1.0 \mathrm{mmol}$ ) following the general procedure afforded $97 \mathrm{mg}(83 \%)$ of the title compound as a pale yellow oil. This material was obtained as a ca. 10:1 mixture of diastereomers as judged by ${ }^{1} \mathrm{H}$ NMR analysis (the crude reaction mixture contained a 5:1 mixture of diastereomers). Data are for the major diastereomer. ${ }^{1} \mathrm{H}$ NMR (500 $\left.\mathrm{MHz}, \mathrm{CDCl}_{3}\right) \delta 8.25(\mathrm{~d}, J=8.5 \mathrm{~Hz}, 1 \mathrm{H}), 7.89(\mathrm{~d}, J=8.0 \mathrm{~Hz}, 1 \mathrm{H}), 7.76(\mathrm{~d}, J=7.5 \mathrm{~Hz}, 1 \mathrm{H})$, 7.58-7.49 (m, $4 \mathrm{H}), 4.45-4.41$ (m, $1 \mathrm{H}), 3.98$ (p, J= 7.0 Hz, $1 \mathrm{H}), 1.88-1.81$ (m, 1 H), 1.79-1.72 (m, 1 $\mathrm{H}), 1.71-1.63(\mathrm{~m}, 2 \mathrm{H}), 1.45(\mathrm{~d}, J=6.5 \mathrm{~Hz}, 3 \mathrm{H}), 1.30(\mathrm{~s}, 3 \mathrm{H}), 1.26(\mathrm{~s}, 3 \mathrm{H}) ;{ }^{13} \mathrm{C} \mathrm{NMR}(125 \mathrm{MHz}$ $\left.\mathrm{CDCl}_{3}\right) \delta 140.2,134.0,132.2,129.0,126.8,125.9,125.6,125.3,124.0,123.6,81.6,80.8,38.8,37.7$ 28.9, 28.1, 28.0, 15.5; IR (film) $1364 \mathrm{~cm}^{-1}$. Anal calcd for $\mathrm{C}_{18} \mathrm{H}_{22} \mathrm{O}$ : C, 84.99; $\mathrm{H}, 8.72$. Found C, 85.06; H, 8.59.

(士)-(1'R,5R)-5-[1'-(4-tert-Butylphenyl)ethyl]-2,2-dimethyltetrahydrofuran (13a). Reaction of (Z)-2-methylhept-5-en-2-ol (32 mg, $0.25 \mathrm{mmol})$ with 1-bromo-4-tert-butylbenzene (107 mg, 0.5 
mmol) following the general procedure afforded $45 \mathrm{mg}(74 \%)$ of the title compound as a pale yellow oil. This material was obtained as a ca. 5:1 mixture of diastereomers as judged by ${ }^{1} \mathrm{H}$ NMR analysis. Data are for the major diastereomer. ${ }^{1} \mathrm{H}$ NMR $\left(500 \mathrm{MHz}, \mathrm{CDCl}_{3}\right) \delta 7.32(\mathrm{~d}, J=8.5 \mathrm{~Hz}, 2 \mathrm{H}), 7.12(\mathrm{~d}, J$ $=8.5 \mathrm{~Hz}, 2 \mathrm{H}), 4.04-3.99(\mathrm{~m}, 1 \mathrm{H}), 2.71-2.68(\mathrm{~m}, 1 \mathrm{H}), 1.72-1.68(\mathrm{~m}, 1 \mathrm{H})$ 1.64-1.55 (m, $3 \mathrm{H}), 1.32$ $(\mathrm{d}, J=8.5 \mathrm{~Hz}, 3 \mathrm{H}), 1.31(\mathrm{~m}, 9 \mathrm{H}), 1.23(\mathrm{~s}, 3 \mathrm{H}), 1.21(\mathrm{~s}, 3 \mathrm{H}) ;{ }^{13} \mathrm{C} \mathrm{NMR}\left(125 \mathrm{MHz}, \mathrm{CDCl}_{3}\right) \delta 148.8$, 141.4, 127.4, 124.9, 83.5, 80.7, 45.4, 38.2, 31.3, 31.3, 30.1, 29.1, 28.2, 18.7; IR (film) $1363 \mathrm{~cm}^{-1}$. Anal calcd for $\mathrm{C}_{18} \mathrm{H}_{28} \mathrm{O}: \mathrm{C}, 83.02 ; \mathrm{H}, 10.84$. Found: C, 83.42; H, 10.57.

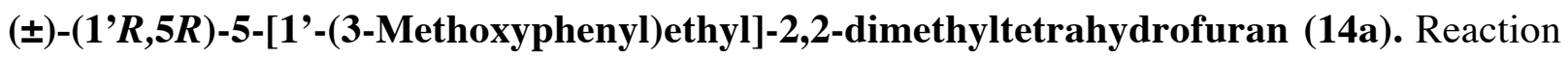
of (Z)-2-methylhept-5-en-2-ol (64 mg, $0.5 \mathrm{mmol})$ with 3-bromoanisole (187 mg, $1.0 \mathrm{mmol})$ following the general procedure afforded $93 \mathrm{mg}(79 \%)$ of the title compound as a pale yellow oil. This material was obtained as a ca. 7:1 mixture of diastereomers as judged by ${ }^{1} \mathrm{H}$ NMR analysis (the crude reaction mixture contained a 5:1 mixture of diastereomers). Data are for the major diastereomer. ${ }^{1} \mathrm{H}$ NMR (500 $\left.\mathrm{MHz}, \mathrm{CDCl}_{3}\right) \delta 7.21(\mathrm{t}, J=7.5 \mathrm{~Hz}, 1 \mathrm{H}), 6.82-6.75(\mathrm{~m}, 3 \mathrm{H}), 4.06-4.02(\mathrm{~m}, 1 \mathrm{H}), 3.81(\mathrm{~s}, 3 \mathrm{H}), 2.71$ $(\mathrm{p}, J=7.5 \mathrm{~Hz}, 1 \mathrm{H}), 1.76-1.71(\mathrm{~m}, 1 \mathrm{H}), 1.63-1.56(\mathrm{~m}, 3 \mathrm{H}), 1.35(\mathrm{~d}, J=7.0 \mathrm{~Hz}, 3 \mathrm{H}), 1.25(\mathrm{~s}, 3 \mathrm{H})$, $1.23(\mathrm{~s}, 3 \mathrm{H}) ;{ }^{13} \mathrm{C}$ NMR $\left(125 \mathrm{MHz}, \mathrm{CDCl}_{3}\right) \delta 159.3,146.2,129.0,120.3,113.8,111.1,83.2,80.7,55.0$, 46.0, 38.2, 30.0, 29.0, 28.1, 18.8; IR (film) $1363 \mathrm{~cm}^{-1}$. Anal calcd for $\mathrm{C}_{15} \mathrm{H}_{22} \mathrm{O}_{2}:$ C, 76.88; $\mathrm{H}, 9.46$. Found: C, 76.71; H, 9.56.

( \pm )-(1'R,5R)-2,2-Dimethyl-5-(1'-o-tolylethyl)tetrahydrofuran (15a). Reaction of (Z)-2methylhept-5-en-2-ol (64 mg, $0.5 \mathrm{mmol})$ with 2-bromotoluene (171 $\mathrm{mg}, 1.0 \mathrm{mmol}$ ) following the general procedure afforded $60 \mathrm{mg}(55 \%)$ of the title compound as a pale yellow oil. This material was obtained as a ca. 3:1 mixture of diastereomers as judged by ${ }^{1} \mathrm{H}$ NMR analysis (the crude reaction mixture contained a 2:1 mixture of diastereomers). Data are for the major diastereomer. ${ }^{1} \mathrm{H}$ NMR (500 
$\left.\mathrm{MHz}, \mathrm{CDCl}_{3}\right) \delta$ 7.30-7.07 (m, $\left.4 \mathrm{H}\right), 4.18-4.13(\mathrm{~m}, 1 \mathrm{H}), 3.02(\mathrm{q}, J=6.5 \mathrm{~Hz}, 1 \mathrm{H}), 2.37(\mathrm{~s}, 3 \mathrm{H})$, 1.81-1.76 (m, $1 \mathrm{H}), 1.69-1.64(\mathrm{~m}, 2 \mathrm{H}), 1.54-1.48(\mathrm{~m}, 1 \mathrm{H}), 1.30(\mathrm{~d}, J=7.0 \mathrm{~Hz}, 3 \mathrm{H}), 1.27$ (s, $6 \mathrm{H})$; ${ }^{13} \mathrm{C} \mathrm{NMR}\left(125 \mathrm{MHz}, \mathrm{CDCl}_{3}\right) \delta 143.2,135.5,130.1,126.3,125.9,125.6,83.6,80.8,40.6,38.3,30.2$, 29.2, 28.2, 20.1, 19.1; IR (film) $1364 \mathrm{~cm}^{-1}$. Anal calcd for $\mathrm{C}_{15} \mathrm{H}_{22} \mathrm{O}: \mathrm{C}, 82.52$; $\mathrm{H}, 10.16$. Found: C, 82.60; H, 10.05 .

(士)-(3aR,6aS,6S)-[4-(2,2-Dimethylhexahydrocyclopenta[b]furan-6-

yl)phenyl]dimethylamine (19). Reaction of 1-cyclopent-2-enyl-2-methylpropan-2-ol (70 mg, 0.5 mmol) with 4-bromo- $N, N$-dimethylaniline $(200 \mathrm{mg}, 1.0 \mathrm{mmol})$ following the general procedure afforded $76 \mathrm{mg}(59 \%)$ of the title compound as a viscous, pale yellow oil. This material was obtained with $\mathrm{dr} \geq 20: 1$ as judged by ${ }^{1} \mathrm{H}$ NMR analysis. ${ }^{1} \mathrm{H}$ NMR $\left(500 \mathrm{MHz}, \mathrm{CDCl}_{3}\right) \delta 7.19(\mathrm{~d}, J=11.0 \mathrm{~Hz}, 2$ H), $6.69(\mathrm{~d}, J=11.0 \mathrm{~Hz}, 2 \mathrm{H}), 4.41(\mathrm{t}, J=7.5 \mathrm{~Hz}, 1 \mathrm{H}), 2.88-2.83(\mathrm{~m}, 7 \mathrm{H}), 2.71-2.64(\mathrm{~m}, 1 \mathrm{H}), 2.07$ $(\mathrm{dd}, J=11.5,15.0 \mathrm{~Hz}, 1 \mathrm{H}), 2.00-1.90(\mathrm{~m}, 1 \mathrm{H}), 1.75-1.61(\mathrm{~m}, 2 \mathrm{H}), 1.51(\mathrm{dd}, J=7.5,15.5 \mathrm{~Hz}, 1 \mathrm{H})$, 1.32-1.27 (m, $4 \mathrm{H}), 1.09$ (s, $3 \mathrm{H}) ;{ }^{13} \mathrm{C} \mathrm{NMR}\left(125 \mathrm{MHz}, \mathrm{CDCl}_{3}\right) \delta 149.2,129.5,128.7,112.7,84.1$, 81.0, 50.6, 47.4, 43.2, 40.8, 32.0, 29.2, 27.7, 25.3; IR (film) $1365 \mathrm{~cm}^{-1}$. Anal calcd for $\mathrm{C}_{17} \mathrm{H}_{25} \mathrm{NO}$ : C, 78.72; H, 9.71; N, 5.40. Found: C, 78.99; H, 9.75; N, 5.36.

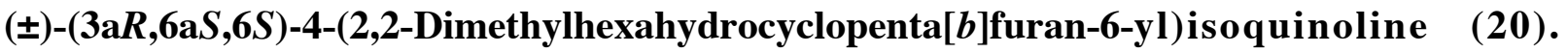
Reaction of 1-cyclopent-2-enyl-2-methylpropan-2-ol (35 mg, $0.25 \mathrm{mmol}$ ) with 4-bromoisoquinoline (104 mg, $0.5 \mathrm{mmol})$ using $5 \mathrm{~mol} \%$ xantphos $(7.2 \mathrm{mg}, 0.0125 \mathrm{mmol})$ in place of $\mathrm{P}(o \text {-tol })_{3}$ following the general procedure afforded $49 \mathrm{mg}(73 \%)$ of the title compound as a viscous, brown oil. This material was obtained with $\mathrm{dr} \geq 20: 1$ as judged by ${ }^{1} \mathrm{H}$ NMR analysis. ${ }^{1} \mathrm{H}$ NMR $\left(500 \mathrm{MHz}, \mathrm{CDCl}_{3}\right) \delta 9.11$ (s, 1 H), $8.53(\mathrm{~s}, 1 \mathrm{H}), 8.09(\mathrm{~d}, J=8.5 \mathrm{~Hz}, 1 \mathrm{H}), 7.94(\mathrm{~d}, J=8.5 \mathrm{~Hz}, 1 \mathrm{H}), 7.68(\mathrm{t}, J=7.5 \mathrm{~Hz}, 1 \mathrm{H}), 7.55(\mathrm{t}$, 
$J=7.0 \mathrm{~Hz}, 1 \mathrm{H}), 4.72(\mathrm{t}, J=6.0 \mathrm{~Hz}, 1 \mathrm{H}), 3.47(\mathrm{dt}, J=5.0,13.0 \mathrm{~Hz}, 1 \mathrm{H}), 3.03(\mathrm{p}, 8.5 \mathrm{~Hz}, 1 \mathrm{H})$, 2.36-2.31 (m, $1 \mathrm{H}), 2.12(\mathrm{dd}, J=9.5,12.5 \mathrm{~Hz}, 1 \mathrm{H}), 1.85-1.76(\mathrm{~m}, 2 \mathrm{H}), 1.68-1.66(\mathrm{~m}, 1 \mathrm{H}), 1.36$ (dd, $J=8.0,12.2 \mathrm{~Hz}, 1 \mathrm{H}), 1.23(\mathrm{~s}, 3 \mathrm{H}), 1.04(\mathrm{~s}, 3 \mathrm{H}) ;{ }^{13} \mathrm{C} \mathrm{NMR}\left(125 \mathrm{MHz}, \mathrm{CDCl}_{3}\right) \delta 151.0,142.8,134.8$, 129.8, 128.6, 128.3, 128.2, 126.2, 122.3, 82.9, 81.4, 47.0, 44.9, 43.4, 31.6, 27.5, 27.0, 25.3; IR (film) $1366 \mathrm{~cm}^{-1}$. Anal calcd for $\mathrm{C}_{18} \mathrm{H}_{21} \mathrm{NO}$ : C, 80.86; H, 7.92; N, 5.24. Found: C, 81.06; H, 7.91; N, 5.12.

(士)-(3aR,7aS,7S)-7-(4-tert-butylphenyl)-2,2-dimethyloctahydrobenzofuran (21). Reaction of 1-cyclohex-2-enyl-2-methylpropan-2-ol (39 mg, $0.25 \mathrm{mmol})$ with 1-bromo-4-tert-butylbenzene (107 $\mathrm{mg}, 0.5 \mathrm{mmol})$ following the general procedure afforded $45 \mathrm{mg}(63 \%)$ of the title compound as a colorless oil. This material was obtained with $\mathrm{dr} \geq 20: 1$ as judged by ${ }^{1} \mathrm{H}$ NMR analysis. ${ }^{1} \mathrm{H}$ NMR $(500$ $\left.\mathrm{MHz}, \mathrm{CDCl}_{3}\right) \delta 7.33-7.29(\mathrm{~m}, 4 \mathrm{H}), 4.09(\mathrm{t}, J=3.0 \mathrm{~Hz}, 1 \mathrm{H}), 2.76(\mathrm{dt}, J=3.0,12.5 \mathrm{~Hz}, 1 \mathrm{H})$, 2.09-2.06 (m, $1 \mathrm{H}), 1.86(\mathrm{dd}, J=7.0,12.2 \mathrm{~Hz}, 1 \mathrm{H}), 1.83-1.78(\mathrm{~m}, 2 \mathrm{H}), 1.69-1.66(\mathrm{~m}, 1 \mathrm{H})$, 1.54-1.53 (m, $1 \mathrm{H}), 1.48-1.37(\mathrm{~m}, 2 \mathrm{H}), 1.35-1.33(\mathrm{~m}, 4 \mathrm{H}), 1.30(\mathrm{~s}, 9 \mathrm{H}), 1.15(\mathrm{~s}, 3 \mathrm{H}) ;{ }^{13} \mathrm{C}$ NMR $\left(125 \mathrm{MHz}, \mathrm{CDCl}_{3}\right) \delta 148.2,141.8,127.6,124.8,78.9,78.2,45.2,44.8,40.6,34.2,31.3,30.6,29.3$, 28.4, 26.9, 25.9; IR (film) $1364 \mathrm{~cm}^{-1}$. Anal calcd for $\mathrm{C}_{20} \mathrm{H}_{30} \mathrm{O}: \mathrm{C}, 83.86 ; \mathrm{H}, 10.56$; Found: C, 83.68; $\mathrm{H}$, 10.30 .

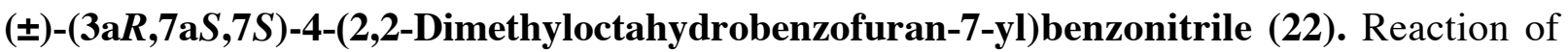
1-cyclohex-2-enyl-2-methylpropan-2-ol (39 mg, $0.25 \mathrm{mmol})$ with 4-bromobenzonitrile (91 $\mathrm{mg}, 0.5$ mmol) following the general procedure using xantphos in place of $\mathrm{P}(\mathrm{o} \text {-tol })_{3}$ afforded $45 \mathrm{mg}(63 \%)$ of the title compound as a pale yellow oil. This material was obtained with $\mathrm{dr} \geq 20: 1$ as judged by ${ }^{1} \mathrm{H}$ NMR analysis. ${ }^{1} \mathrm{H}$ NMR $\left(500 \mathrm{MHz}, \mathrm{CDCl}_{3}\right) \delta 7.55(\mathrm{~d}, J=8.0 \mathrm{~Hz}, 2 \mathrm{H}), 7.47(\mathrm{~d}, J=8.0 \mathrm{~Hz}, 2 \mathrm{H}), 4.00$ $(\mathrm{t}, \quad J=3.0 \mathrm{~Hz}, 1 \mathrm{H}), 2.83(\mathrm{dt}, J=3.5,12.5 \mathrm{~Hz}, 1 \mathrm{H}), 2.12-2.07(\mathrm{~m}, 1 \mathrm{H}), 1.89-1.79(\mathrm{~m}, 3 \mathrm{H})$, 
1.64-1.55 (m, $2 \mathrm{H}), 1.51-1.40(\mathrm{~m}, 2 \mathrm{H}), 1.37-1.33(\mathrm{~m}, 4 \mathrm{H}), 1.10(\mathrm{~s}, 3 \mathrm{H}) ;{ }^{13} \mathrm{C}$ NMR $(125 \mathrm{MHz}$, $\left.\mathrm{CDCl}_{3}\right) \delta 150.4,131.7,129.0,119.3,109.5,79.2,77.5,45.7,45.1,40.5,30.5,29.2,28.2,26.4,25.5 ; \mathrm{IR}$ (film) $1365 \mathrm{~cm}^{-1}$. Anal calcd for $\mathrm{C}_{17} \mathrm{H}_{21} \mathrm{NO}$ : C, 79.96; H, 8.29; N, 5.49. Found: $\mathrm{C}, 79.89 ; \mathrm{H}, 8.24 ; \mathrm{N}$, 5.58 .

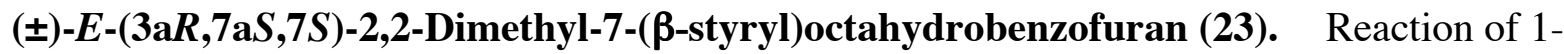
cyclohex-2-enyl-2-methylpropan-2-ol (77 mg, $0.5 \mathrm{mmol})$ with $\beta$-bromostyrene (183 $\mathrm{mg}, 1.0 \mathrm{mmol})$ following the general procedure afforded $91 \mathrm{mg}(71 \%)$ of the title compound as a brown oil. This material was obtained with $\mathrm{dr} \geq 20: 1$ as judged by ${ }^{1} \mathrm{H}$ NMR analysis. ${ }^{1} \mathrm{H}$ NMR $\left(500 \mathrm{MHz}, \mathrm{CDCl}_{3}\right) \delta$ $7.38(\mathrm{~d}, J=7.0 \mathrm{~Hz}, 2 \mathrm{H}), 7.28(\mathrm{t}, J=7.5 \mathrm{~Hz}, 2 \mathrm{H}), 7.17(\mathrm{t}, J=7.5 \mathrm{~Hz}, 1 \mathrm{H}), 6.41-6.40(\mathrm{~m}, 2 \mathrm{H}), 3.90$ $(\mathrm{t}, J=3.5 \mathrm{~Hz}, 1 \mathrm{H}), 2.42-2.38(\mathrm{~m}, 1 \mathrm{H}), 2.15-1.96(\mathrm{~m}, 1 \mathrm{H}), 1.87(\mathrm{dd}, J=7.5,12.5 \mathrm{~Hz}, 1 \mathrm{H})$, 1.74-1.72 (m, $1 \mathrm{H}), 1.56-1.48(\mathrm{~m}, 4 \mathrm{H}), 1.37$ (s, $3 \mathrm{H}), 1.34-1.28(\mathrm{~m}, 2 \mathrm{H}), 1.19(\mathrm{~s}, 3 \mathrm{H}) ;{ }^{13} \mathrm{C}$ NMR $\left(125 \mathrm{MHz}, \mathrm{CDCl}_{3}\right) \delta 137.9,134.0,128.8,128.3,126.6,126.1,78.8,78.4,45.3,43.5,39.9,30.6,29.3$, 28.3, 26.5, 25.1; IR (film) $1365 \mathrm{~cm}^{-1}$. Anal calcd for $\mathrm{C}_{18} \mathrm{H}_{24} \mathrm{O}$ : C, 84.32; H, 9.44;. Found: C, 84.39; H, 9.34 .

( \pm )-(1R,6S)-6-(4-tert-Butylphenyl)-2',2'-dimethyl-1-oxaspiro[4.5] decane (24). Reaction of 4-cyclohex-1-enyl-2-methylbutan-2-ol (42 mg, $0.25 \mathrm{mmol}$ ) with 1-bromo-4-tert-butylbenzene (107 $\mathrm{mg}, 0.5 \mathrm{mmol})$ following the general procedure afforded $49 \mathrm{mg}(65 \%)$ of the title compound as a pale yellow oil. This material was obtained with $\mathrm{dr} \geq 20: 1$ as judged by ${ }^{1} \mathrm{H}$ NMR analysis. ${ }^{1} \mathrm{H}$ NMR (500 $\left.\mathrm{MHz}, \mathrm{CDCl}_{3}\right) \delta 7.29-7.25(\mathrm{~m}, 4 \mathrm{H}), 2.43(\mathrm{dd}, J=3.5,13.0 \mathrm{~Hz}, 1 \mathrm{H}), 2.08(\mathrm{dq}, J=3.7,13.0 \mathrm{~Hz}, 1 \mathrm{H})$, 1.88-1.76 (m, 4 H), 1.63-1.58 (m, 3 H), 1.52-1.37 (m, 3 H), 1.33 (s, 9 H), 1.21 (s, 3 H), 0.95 (s, 3 H), 0.79-0.74 (m, $1 \mathrm{H}) ;{ }^{13} \mathrm{C} \mathrm{NMR}\left(125 \mathrm{MHz}, \mathrm{CDCl}_{3}\right) \delta$ 148.8, 140.3, 129.5, 124.0, 84.2, 80.9, 52.2, 40.5, 
37.4, 36.2, 34.3, 31.4, 29.8, 29.6, 28.5, 26.5, 22.5; IR (film) $1362 \mathrm{~cm}^{-1}$. Anal calcd for $\mathrm{C}_{21} \mathrm{H}_{32} \mathrm{O}: \mathrm{C}$, 83.94; H, 10.73;. Found: C, 84.32; H, 10.88.

$( \pm)-(1 R, 6 S)$-[4-(2',2'-Dimethyl-1-oxaspiro[4.5]dec-6-yl)phenyl]phenylmethanone (25). Reaction of 4-cyclohex-1-enyl-2-methylbutan-2-ol (42 mg, $0.25 \mathrm{mmol})$ with 4-bromobenzophenone (131 $\mathrm{mg}, 0.5 \mathrm{mmol}$ ) following the general procedure afforded $30 \mathrm{mg}(34 \%)$ of the title compound as a pale yellow oil. This material was obtained with $\mathrm{dr} \geq 20: 1$ as judged by ${ }^{1} \mathrm{H}$ NMR analysis. ${ }^{1} \mathrm{H}$ NMR $\left(500 \mathrm{MHz}, \mathrm{CDCl}_{3}\right) \delta 7.78(\mathrm{~d}, J=7.5 \mathrm{~Hz}, 2 \mathrm{H}), 7.72(\mathrm{~d}, J=8.5 \mathrm{~Hz}, 2 \mathrm{H}), 7.57(\mathrm{t}, J=7.5 \mathrm{~Hz}, 1 \mathrm{H}), 7.47$ (t, $J=7.5 \mathrm{~Hz}, 2 \mathrm{H}), 7.43(\mathrm{~d}, J=7.0 \mathrm{~Hz}, 2 \mathrm{H}), 2.54(\mathrm{dd}, J=3.5,12.7 \mathrm{~Hz}, 1 \mathrm{H}), 2.09$ (dq, $J=4.0,13.0$ Hz, $1 \mathrm{H}), 1.89-1.83$ (m, $1 \mathrm{H}), 1.83-1.74$ (m, $3 \mathrm{H}), 1.66-1.59$ (m, $3 \mathrm{H}), 1.59-1.51$ (m, $1 \mathrm{H}), 1.45-1.33$ (m, 2 H), 1.20 (s, $3 \mathrm{H}), 0.94$ (s, $3 \mathrm{H}), 0.86-0.81(\mathrm{~m}, 1 \mathrm{H}) ;{ }^{13} \mathrm{C}$ NMR $\left(125 \mathrm{MHz}, \mathrm{CDCl}_{3}\right) \delta$ 196.7, 148.8, $137.9,135.3,132.0,129.9,129.3,128.1,83.4,81.0,52.9,40.2,37.4,36.1,29.8,29.5,28.6,26.2,22.3$ (two aromatic signals are incidentally equivalent); IR (film) $1659,1363 \mathrm{~cm}^{-1}$. Anal calcd for $\mathrm{C}_{24} \mathrm{H}_{28} \mathrm{O}_{2}$ : C, 82.72; H, 8.10. Found: C, 82.72; H, 8.17.

( \pm )-E-(1R,6S)-2',2'-Dimethyl-6-( $\beta$-styryl)-1-oxaspiro[4.5]decane (26). Reaction of 4cyclohex-1-enyl-2-methylbutan-2-ol (42 mg, $0.25 \mathrm{mmol}$ ) with $\beta$-bromostyrene (92 mg, $0.5 \mathrm{mmol}$ ) following the general procedure afforded $43 \mathrm{mg}(64 \%)$ of the title compound as a brown oil. This material was obtained with $\mathrm{dr} \geq 20: 1$ as judged by ${ }^{1} \mathrm{H}$ NMR analysis. ${ }^{1} \mathrm{H}$ NMR $\left(500 \mathrm{MHz}, \mathrm{CDCl}_{3}\right) \delta$ $7.35(\mathrm{~d}, J=9.0 \mathrm{~Hz}, 2 \mathrm{H}), 7.29(\mathrm{t}, J=8.0 \mathrm{~Hz}, 2 \mathrm{H}), 7.18(\mathrm{t}, J=7.5 \mathrm{~Hz}, 1 \mathrm{H}), 6.35(\mathrm{~d}, J=16.0 \mathrm{~Hz}, 1$ H), $6.28(\mathrm{dd}, J=8.5,16.2 \mathrm{~Hz}, 1 \mathrm{H}), 2.08-2.02(\mathrm{~m}, 2 \mathrm{H}), 1.81-1.59(\mathrm{~m}, 8 \mathrm{H}), 1.54-1.49(\mathrm{~m}, 2 \mathrm{H})$, 1.33-1.28 (m, $1 \mathrm{H}), 1.26$ (s, $3 \mathrm{H}), 1.24(\mathrm{~s}, 3 \mathrm{H}) ;{ }^{13} \mathrm{C} \mathrm{NMR}\left(125 \mathrm{MHz}, \mathrm{CDCl}_{3}\right) \delta 137.9,133.2,129.8$, 128.5, 126.7, 125.9, 83.8, 80.9, 50.3, 39.2, 37.9, 36.3, 30.0, 29.7, 28.9, 25.1, 22.4; IR (film) $1363 \mathrm{~cm}^{-1}$. 
Anal calcd for $\mathrm{C}_{19} \mathrm{H}_{26} \mathrm{O}$ : C, 84.39; H, 9.69. Found: C, 84.50; H, 10.03.

\section{Assignment of Stereochemistry}

\section{1',2-Disubstituted Tetrahydrofurans}

The stereochemical assignment of $\mathbf{4 a}$ and $\mathbf{1 0 a}$ has been previously described in the supporting information for the preliminary communication of these studies. ${ }^{1}$ The stereochemistry of $\mathbf{1 1 a}-\mathbf{1 5 a}$ was assigned by comparison of ${ }^{1} \mathrm{H}$ and ${ }^{13} \mathrm{C}$ NMR spectra obtained for $\mathbf{1 1 a}-\mathbf{1 5 a}$ to the NMR data obtained for the major and minor diastereomers of $\mathbf{4 a}$ and $\mathbf{1 0 a}$.

\section{2-Ethyl-3-aryl Tetrahydrofurans (Regioisomeric Side Products)}

The stereochemistry of $\mathbf{4 b}$ was assigned on the basis of the nOe signals shown below. The stereochemistry of the analogous regioisomeric products $\mathbf{1 1 b}-\mathbf{1 5}$ was assigned based on analogy to $\mathbf{4 b}$.

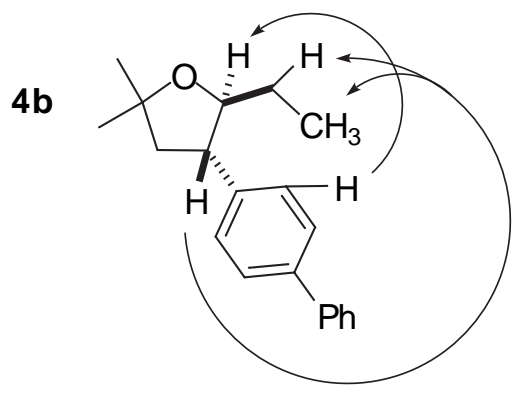

\section{Octahydrocyclopenta $[b]$ furans}

The stereochemical assignment of $\mathbf{2}$ has been previously has been previously described in the supporting information for the preliminary communication of these studies. ${ }^{1}$ The relative stereochemistry of $\mathbf{1 9}$ and 20 was assigned by comparison of ${ }^{1} \mathrm{H}$ and ${ }^{13} \mathrm{C}$ NMR spectra obtained for 19-20 to the NMR data obtained for 2. 


\section{Octahydrobenzofurans}

The relative stereochemistry of $\mathbf{2 1}$ was assigned on the basis of nOe signals between the C3a, C7a, and C7 hydrogens as illustrated below.

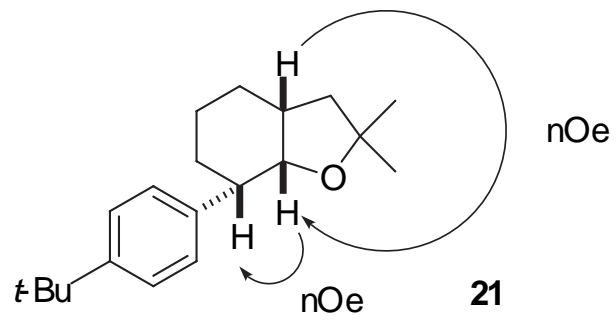

The relative stereochemistry of the octahydrobenzofuran products $\mathbf{2 2}$ and $\mathbf{2 3}$ were assigned based on analogy with this molecule.

\section{Oxaspiro[4.5]decanes}

The relative stereochemistry of $\mathbf{2 4}$ was determined by comparing the ${ }^{1} \mathrm{H}$ NMR spectrum of this compound to that obtained from a different sample of $\mathbf{2 4}$ prepared using the synthetic route illustrated in Scheme 9. The relative stereochemistry of $\mathbf{2 5}$ and $\mathbf{2 6}$ was assigned based on analogy with this molecule.

Scheme 9. Alternative Synthesis of 24
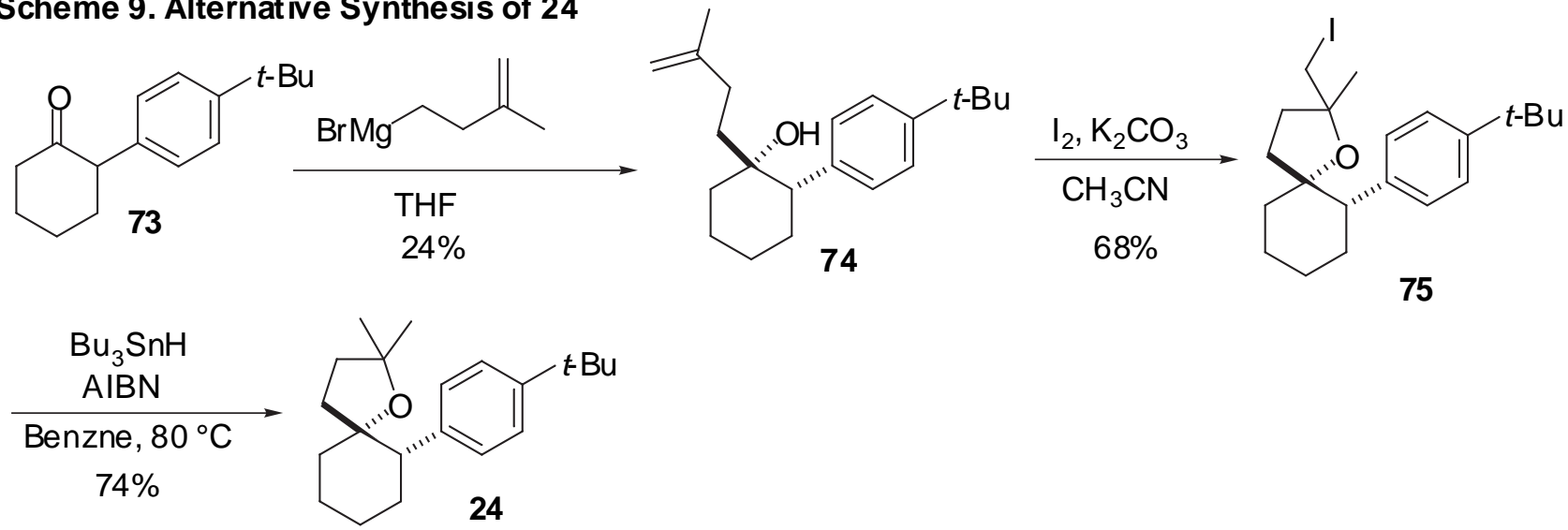


\section{$( \pm)-(1 R, 6 S)-6-(4-t e r t-B u t y l p h e n y l)-2 ', 2 '-d i m e t h y l-1-o x a s p i r o[4.5] d e c a n e$ (Prepared as shown}

above in Scheme 9) (24). A flame dried flask was cooled under a stream of argon and charged with Mg turnings (1.08 g, $44.8 \mathrm{mmol})$. THF $(11 \mathrm{~mL})$ was added, and the suspension was cooled to $0{ }^{\circ} \mathrm{C}$. Neat 1-bromo-3-methylbut-3-ene ${ }^{10}(1.67 \mathrm{~g}, 11.2 \mathrm{mmol})$ was added dropwise to the suspension, and the resulting solution was warmed to $\mathrm{rt}$ with stirring for $1.5 \mathrm{~h}$. The reaction mixture was transferred to a dry, argon filled flask via cannula and additional THF $(11 \mathrm{~mL})$ was added by syringe. A solution of 2(4-tert-butylphenyl)cyclohexanone $(73)^{11}(1.30 \mathrm{~g}, 5.60 \mathrm{mmol})$ in THF $(4 \mathrm{~mL})$ was added, the resulting solution was stirred at $\mathrm{rt}$ for $8 \mathrm{~h}$, and then saturated aqueous $\mathrm{NH}_{4} \mathrm{Cl}(20 \mathrm{~mL})$ was added dropwise. The resulting mixture was diluted with ethyl acetate $(40 \mathrm{~mL})$ and the aqueous layer was removed and extracted with ethyl acetate $(2 \times 20 \mathrm{~mL})$. The organic layers were combined and dried over anhydrous $\mathrm{Na}_{2} \mathrm{SO}_{4}$, decanted, and concentrated in vacuo. The crude material was obtained as an 8:1 mixture of diastereomers as determined by ${ }^{1} \mathrm{H}$ NMR analysis. The diastereomers were separated by flash chromatography on silica gel (19:1 hexanes/ethyl acetate) to afford $804 \mathrm{mg}(24 \%)$ of (74). The relative stereochemistry was assigned by comparison of NMR spectral data to those previously reported for the related compound $(1 S, 2 R)$-1-allyl-2-phenylcyclohexanol. ${ }^{12}{ }^{1} \mathrm{H}$ NMR (500 $\mathrm{MHz}$, $\left.\mathrm{CDCl}_{3}\right) \delta 7.30(\mathrm{~d}, J=8 \mathrm{~Hz}, 2 \mathrm{H}), 7.18(\mathrm{~d}, J=8 \mathrm{~Hz}, 2 \mathrm{H}), 4.61(\mathrm{~s}, 1 \mathrm{H}), 4.55(\mathrm{~s}, 1 \mathrm{H}), 2.55(\mathrm{dd}, J=3.2$, $13.0 \mathrm{~Hz}, 1 \mathrm{H}), 2.12-2.01(\mathrm{~m}, 2 \mathrm{H}), 1.99-1.91(\mathrm{~m}, 1 \mathrm{H}), 1.87-1.82(\mathrm{~m}, 2 \mathrm{H}), 1.81(\mathrm{~s}, 1 \mathrm{H}), 1.74-1.67$ (m, $1 \mathrm{H}), 1.62(\mathrm{~m}, 5 \mathrm{H}), 1.46-1.37$ (m, $4 \mathrm{H}), 1.35$ (s, $9 \mathrm{H})$.

A flame dried flask was cooled under a stream of argon and charged with (74) (310 mg, 1.03 $\mathrm{mmol})$, potassium carbonate $(184 \mathrm{mg}, 1.34 \mathrm{mmol})$, and acetonitrile $(13 \mathrm{~mL})$. Iodine (1.72 $\mathrm{g}, 6.78$ mmol) was added and the solution was stirred at $\mathrm{rt}$ until the starting material had been completely consumed as judged by TLC analysis $(\sim 3 \mathrm{~h})$. Water $(20 \mathrm{~mL})$ and methylene chloride $(40 \mathrm{~mL})$ were added to the reaction mixture and the layers were separated. The organic layer washed with water $(2 \mathrm{x}$ 
$20 \mathrm{~mL}$ ) and saturated aqueous $\mathrm{Na}_{2} \mathrm{SO}_{3}(1 \times 20 \mathrm{~mL})$, and then was dried over anhydrous $\mathrm{MgSO}_{4}$, decanted, and concentrated in vacuo. The crude product was purified by flash chromatography on silica gel (49:1 hexanes/ethyl acetate) to afford $299 \mathrm{mg}(68 \%)$ of $\mathbf{7 5}$ as a 2:1 mixture of diastereomers as judged by ${ }^{1} \mathrm{H}$ NMR analysis; data is for the mixture. ${ }^{1} \mathrm{H}$ NMR $\left(400 \mathrm{MHz}, \mathrm{CDCl}_{3}\right) \delta 7.31(\mathrm{~d}, J=8.0$ Hz, 2 H), $7.18(\mathrm{~d}, J=8.0 \mathrm{~Hz}, 2 \mathrm{H}), 3.25(\mathrm{~d}, J=9.6 \mathrm{~Hz}, 0.67 \mathrm{H}), 3.18(\mathrm{~d}, J=9.6 \mathrm{~Hz}, 0.67 \mathrm{H}), 2.84(\mathrm{~d}$, $J=9.6 \mathrm{~Hz}, 0.33 \mathrm{H}), 2.61(\mathrm{~d}, J=9.2 \mathrm{~Hz}, 0.33 \mathrm{H}), 2.45-2.37(\mathrm{~m}, 1 \mathrm{H}), 2.11-2.00(\mathrm{~m}, 1 \mathrm{H}), 1.88-1.67$ (m, $4 \mathrm{H}), 1.60-1.44(\mathrm{~m}, 3 \mathrm{H}), 1.44-1.43(\mathrm{~m}, 1 \mathrm{H}), 1.34-1.31(\mathrm{~m}, 1 \mathrm{H}), 1.30(\mathrm{~s}, 9 \mathrm{H}), 1.25-1.22$ (m, 1 H), $1.06(\mathrm{~s}, 3 \mathrm{H}), 0.88-0.81(\mathrm{~m}, 1 \mathrm{H})$.

A flame dried flask was cooled under a stream of argon and charged with (75) (299 mg, 0.70 mmol), AIBN (5.7 mg, $0.035 \mathrm{mmol})$, and benzene (2.3 mL). Tributyltin hydride (235 mg, $0.84 \mathrm{mmol})$ was added, and the reaction mixture was heated to reflux. The starting material was consumed after $3 \mathrm{~h}$ as judged by capillary GC analysis, and the reaction mixture was concentrated in vacuo. The crude product was dissolved in diethyl ether $(10 \mathrm{~mL})$ and treated with a solution saturated aqueous potassium fluoride $(10 \mathrm{~mL})$. The resulting biphasic solution was stirred at $\mathrm{rt}$ for $2 \mathrm{~h}$, then the aqueous layer was separated and extracted with diethyl ether $(2 \times 20 \mathrm{~mL})$. The organic layers were combined and dried over anhydrous $\mathrm{MgSO}_{4}$, decanted, and concentrated in vacuo. The crude product was purified by flash chromatography on silica gel (19:1 hexanes/ethyl acetate) to afford $155 \mathrm{mg}(74 \%)$ of $( \pm)-(1 R, 6 S)-6-(4-$ tert-Butylphenyl)-2',2'-dimethyl-1-oxaspiro[4.5]decane (24). ${ }^{1} \mathrm{H}$ NMR (500 MHz, $\left.\mathrm{CDCl}_{3}\right) \delta$ 7.29-7.25 $(\mathrm{m}, 4 \mathrm{H}), 2.43(\mathrm{dd}, J=3.5,13.0 \mathrm{~Hz}, 1 \mathrm{H}), 2.08(\mathrm{dq}, J=3.7,13.0 \mathrm{~Hz}, 1 \mathrm{H}), 1.88-1.76(\mathrm{~m}, 4 \mathrm{H})$, $1.63-1.58(\mathrm{~m}, 3 \mathrm{H}), 1.52-1.37(\mathrm{~m}, 3 \mathrm{H}), 1.33(\mathrm{~s}, 9 \mathrm{H}), 1.21(\mathrm{~s}, 3 \mathrm{H}), 0.95(\mathrm{~s}, 3 \mathrm{H}), 0.79-0.74(\mathrm{~m}, 1 \mathrm{H})$. 


\section{Deuterium Labeling Studies (eq 3-5)}

(E)-5-Deuterio-2-methylhept-5-en-2-ol (27). A flame dried flask equipped with a reflux condenser was cooled under a stream of argon and charged with $\mathrm{LiAlD}_{4}(50 \mathrm{~mL}, 50 \mathrm{~mL}, 1.0 \mathrm{M}$ in THF). But-3-yn-2-ol (1.75 g, $25 \mathrm{mmol})$ was added and the mixture was heated to reflux for $20 \mathrm{~h}$. The reaction was monitored by ${ }^{1} \mathrm{H}$ NMR, and upon consumption of the starting material the reaction was carefully quenched by slowly adding water $(2.2 \mathrm{~mL}), \mathrm{NaOH}(2.2 \mathrm{~mL}, 10 \mathrm{M})$, and additional water $(6.6$ $\mathrm{mL}$ ). The resulting suspension was decanted and the solvent was removed by fractional distillation to afford 3-deuteriobut-3-en-2-ol as a 2:1 mixture of the title compound and THF. This material was used without further purification. ${ }^{1} \mathrm{H}$ NMR $\left(400 \mathrm{MHz}, \mathrm{CDCl}_{3}\right) \delta 5.21-5.19(\mathrm{~m}, 1 \mathrm{H}), 5.06-5.05(\mathrm{~m}, 1 \mathrm{H})$, $4.30(\mathrm{q}, J=6.0 \mathrm{~Hz}, 1 \mathrm{H}), 1.52(\mathrm{~s}, 1 \mathrm{H}), 1.27(\mathrm{~d}, J=6.4 \mathrm{~Hz}, 3 \mathrm{H})$.

The crude 3-deuteriobut-3-en-2-ol described above was treated with triethyl orthoacetate using general procedure 2 to afford $391 \mathrm{mg}$ (7\% over two steps) of (E)-4-deuteriohex-4-enoic acid ethyl ester. ${ }^{1} \mathrm{H}$ NMR (400 MHz, $\left.\mathrm{CDCl}_{3}\right) \delta 5.48-5.45(\mathrm{~m}, 1 \mathrm{H}), 4.12(\mathrm{q}, J=6.0 \mathrm{~Hz}, 2 \mathrm{H}), 2.36-2.30(\mathrm{~m}, 2 \mathrm{H})$, 2.30-2.27 (m, $2 \mathrm{H}), 1.63(\mathrm{~d}, J=6.8 \mathrm{~Hz}, 3 \mathrm{H}), 1.24(\mathrm{t}, J=6.0 \mathrm{~Hz}, 3 \mathrm{H})$.

(E)-4-Deuteriohex-4-enoic acid ethyl ester (391 mg, $2.73 \mathrm{mmol}$ ) was treated with $\mathrm{MeMgBr}$ (2.7 $\mathrm{mL}, 8.1 \mathrm{mmol}, 3.0 \mathrm{M}$ in diethyl ether) according to general procedure 1 to afford $200 \mathrm{mg}$ (57\%) of the title compound as a colorless oil. This material was found to contain $\geq 95 \%$ D incorporation at C5 as judged by ${ }^{1} \mathrm{H}$ and ${ }^{13} \mathrm{C}$ NMR and MS analysis. ${ }^{1} \mathrm{H}$ NMR (400 MHz, $\left.\mathrm{CDCl}_{3}\right) \delta 5.46-5.44(\mathrm{~m}, 1 \mathrm{H})$, 2.08-2.04 (m, $2 \mathrm{H}), 1.64(\mathrm{~d}, J=5.2 \mathrm{~Hz}, 3 \mathrm{H}), 1.55-1.51(\mathrm{~m}, 2 \mathrm{H}), 1.28(\mathrm{~s}, 1 \mathrm{H}), 1.21(\mathrm{~s}, 6 \mathrm{H}) ;{ }^{2} \mathrm{H}$ NMR $\left(83 \mathrm{MHz}, \mathrm{CHCl}_{3}\right) \delta 5.42(\mathrm{~s}, 1 \mathrm{D}) ;{ }^{13} \mathrm{C} \mathrm{NMR}\left(125 \mathrm{MHz}, \mathrm{CDCl}_{3}\right) \delta 131.0\left(\mathrm{t}, J_{\mathrm{CD}}=22.7 \mathrm{~Hz}\right), 124.6,70.8$, 43.3, 29.1, 27.3, 17.7; IR (film) $3359 \mathrm{~cm}^{-1}$; MS (EI) $\mathrm{m} / z 111.1160$ (111.1157 calcd for $\mathrm{C}_{8} \mathrm{H}_{15} \mathrm{DO}$, $\left.\mathrm{M}-\mathrm{H}_{2} \mathrm{O}\right)$. 


\section{Palladium-Catalyzed Reaction of (E)-5-Deuterio-2-methylhept-5-en-2-ol with 4-}

Bromobiphenyl (eq 3). Reaction of $32 \mathrm{mg}(0.25 \mathrm{mmol})(E)$-5-deuterio-2-methylhept-5-en-2-ol with 4bromobiphenyl (117 mg, $0.5 \mathrm{mmol}$ ) according to the general procedure afforded $35 \mathrm{mg}(50 \%)$ of deuterated $( \pm)-(1 ' S, 5 R)-5-[1$ '-(4-phenyl)phenylethyl]-2,2-dimethyltetrahydrofuran-D-1 The material was isolated as a ca. 79:17:4 mixture of $\mathbf{2 8 : 2 9 : 3 0}$ as judged by ${ }^{1} \mathrm{H}$ NMR analysis; all isomers contained a single deuterium atom as judged by MS analysis. The major diastereomer $\mathbf{2 8}$ was found to be deuterated at C5 (94\%D), the minor diastereomer 29 was mainly deuterated at $\mathrm{C} 1{ }^{\prime}(62 \% \mathrm{D})$, and the minor 3-aryl regioisomer $\mathbf{3 0}$ was deuterated at $\mathrm{C}^{\prime}$ '. Complete data are given for the major diastereomer (28); selected data are shown below for the minor isomers (Table 3). ${ }^{1} \mathrm{H}$ NMR (400 $\left.\mathrm{MHz}, \mathrm{CDCl}_{3}\right) \delta$ $7.65(\mathrm{~d}, J=7.0 \mathrm{~Hz}, 2 \mathrm{H}), 7.59(\mathrm{~d}, J=8.0 \mathrm{~Hz}, 2 \mathrm{H}), 7.48-7.35(\mathrm{~m}, 5 \mathrm{H}), 2.94(\mathrm{q}, J=6.8 \mathrm{~Hz}, 1 \mathrm{H})$, 1.92-1.86 (m, $1 \mathrm{H}), 1.79-1.72(\mathrm{~m}, 1 \mathrm{H}), 1.68-1.56(\mathrm{~m}, 2 \mathrm{H}), 1.31(\mathrm{~d}, J=7.2 \mathrm{~Hz}, 3 \mathrm{H}), 1.22(\mathrm{~s}, 6 \mathrm{H}) ;{ }^{2} \mathrm{H}$ NMR (83 MHz, $\left.\mathrm{CHCl}_{3}\right) \delta 4.16$ (s, $\left.1 \mathrm{D}\right) ;{ }^{13} \mathrm{C}$ NMR (100 MHz, $\left.\mathrm{CDCl}_{3}\right) \delta 143.3,141.1,138.9,128.6$, $128.5,126.9,126.8,126.7,82.5\left(\mathrm{t}, J_{\mathrm{CD}}=19.2 \mathrm{~Hz}\right), 80.5,43.7,38.4,28.7,28.3,28.0,16.4$; IR (film) $1363 \mathrm{~cm}^{-1}$. MS (EI) $\mathrm{m} / z 281.1886$ (281.1889 calcd for $\left.\mathrm{C}_{20} \mathrm{H}_{23} \mathrm{DO}\right)$.

Table 3. Selected NMR Data for the mixture of 28, 29, and 30

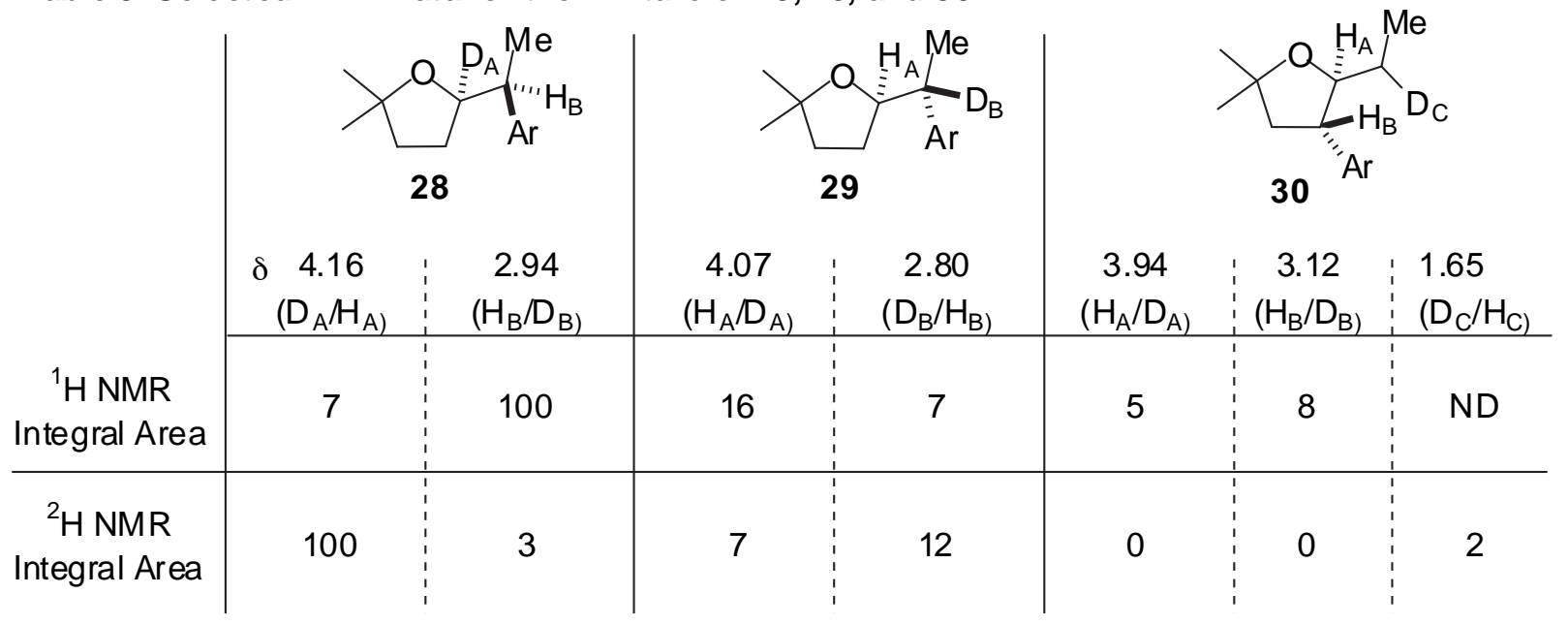


(E)-6-Deuterio-2-methylhept-5-en-2-ol (31). A flame-dried flask was cooled under a stream of argon and charged with $\mathrm{LiAlD}_{4}(1.41 \mathrm{~g}, 35 \mathrm{mmol})$ and diethyl ether $(20 \mathrm{~mL})$. The mixture was cooled to $0^{\circ} \mathrm{C}$ and methyl vinyl ketone $(2.03 \mathrm{~g}, 29 \mathrm{mmol})$ in diethyl ether $(5 \mathrm{~mL})$ was added dropwise. The solution was warmed to rt over $8 \mathrm{~h}$ and monitored by ${ }^{1} \mathrm{H}$ NMR. Upon consumption of the starting material the reaction was carefully quenched by slowly adding water $(2 \mathrm{~mL}), \mathrm{NaOH}(2 \mathrm{~mL}, 10 \mathrm{M})$, and additional water $(5 \mathrm{~mL})$. The resulting suspension was decanted and the solvent was removed by fractional distillation to provide $1.37 \mathrm{~g}(65 \%)$ of 2-deuteriobut-3-en-2-ol as a clear liquid. ${ }^{1} \mathrm{H}$ NMR $\left(400 \mathrm{MHz}, \mathrm{CDCl}_{3}\right) \delta 5.86(\mathrm{dd}, J=11.6,16.8 \mathrm{~Hz}, 1 \mathrm{H}), 5.16(\mathrm{dd}, J=1.6,17.2 \mathrm{~Hz}, 1 \mathrm{H}), 5.01(\mathrm{dd}, J=$ 1.6, $10.8 \mathrm{~Hz}, 1 \mathrm{H}), 1.42$ (s, $1 \mathrm{H}), 1.20$ (s, $3 \mathrm{H})$.

2-Deuteriobut-3-en-2-ol (1.20 g, $16 \mathrm{mmol})$ was treated with triethylorthoacetate according to general procedure 2 to afford $1.07 \mathrm{~g}(46 \%)$ of $(E)-5$-deuteriohex-4-enoic acid ethyl ester as a pale

yellow liquid. ${ }^{1} \mathrm{H}$ NMR (500 MHz, $\left.\mathrm{CDCl}_{3}\right) \delta 5.42-5.39(\mathrm{~m}, 1 \mathrm{H}), 4.12(\mathrm{q}, J=7.0 \mathrm{~Hz}, 2 \mathrm{H}), 2.36-2.32$ (m, $2 \mathrm{H}), 2.31-2.27(\mathrm{~m}, 2 \mathrm{H}), 1.63(\mathrm{~s}, 3 \mathrm{H}), 1.24(\mathrm{t}, J=7.0 \mathrm{~Hz}, 3 \mathrm{H})$.

(E)-5-Deuteriohex-4-enoic acid ethyl ester (1.07 g, $7.5 \mathrm{mmol})$ was treated with $\mathrm{MeMgBr}(7.5$ $\mathrm{mL}, 22.5 \mathrm{mmol}, 3.0 \mathrm{M}$ in diethyl ether) according to general procedure 1 to afford $460 \mathrm{mg}(47 \%)$ of the title compound as a clear oil. ${ }^{1} \mathrm{H}$ NMR $\left(400 \mathrm{MHz}, \mathrm{CDCl}_{3}\right) \delta 5.42-5.35(\mathrm{~m}, 1 \mathrm{H}), 2.03-1.99(\mathrm{~m}, 2$ $\mathrm{H}), 1.78(\mathrm{~s}, 1 \mathrm{H}), 1.58(\mathrm{~s}, 3 \mathrm{H}), 1.49-1.45(\mathrm{~m}, 2 \mathrm{H}), 1.15(\mathrm{~s}, 6 \mathrm{H}) ;{ }^{2} \mathrm{H}$ NMR $\left(83 \mathrm{MHz}, \mathrm{CHCl}_{3}\right) \delta 5.45(\mathrm{~s}$, $1 \mathrm{D}) ;{ }^{13} \mathrm{C}$ NMR $\left(100 \mathrm{MHz}, \mathrm{CDCl}_{3}\right) \delta 131.2,124.3\left(\mathrm{t}, J_{\mathrm{CD}}=17.8 \mathrm{~Hz}\right), 70.7,43.3,29.0,27.4,17.6$; IR (film) $3368 \mathrm{~cm}^{-1}$; MS (EI) $m / z 111.1153$ (111.1157 calcd for $\mathrm{C}_{8} \mathrm{H}_{15} \mathrm{DO}, \mathrm{M}-\mathrm{H}_{2} \mathrm{O}$ ).

\section{Palladium-Catalyzed Reaction of $(E)$-6-Deuterio-2-methylhept-5-en-2-ol with 4-} bromobiphenyl (eq 4). Reaction of $64.5 \mathrm{mg}(0.5 \mathrm{mmol})(E)-6$-deuterio-2-methylhept-5-en-2-ol with 4-bromobiphenyl (233 mg, $1.0 \mathrm{mmol})$ according to the general procedure afforded $93 \mathrm{mg}(66 \%)$ of 
$( \pm)-(1 ’ S, 5 R)-5-[1$ '-(4-Phenyl)phenylethyl]-2,2-dimethyltetrahydrofuran-D-1. The material was isolated as a ca. 75:20:5 mixture of 32:33:30 as judged by ${ }^{1} \mathrm{H}$ NMR analysis; all isomers contained a single deuterium atom as judged by MS analysis. The major diastereomer $\mathbf{3 2}$ was found to be deuterated at $\mathrm{C} 1$ ' $(95 \% \mathrm{D})$, the minor diastereomer $\mathbf{3 3}$ was mainly deuterated at $\mathrm{C} 5(78 \% \mathrm{D})$, and the minor 3-aryl regioisomer 32 was deuterated at $\mathrm{C}^{\prime}$ '. Complete data are given for the major diastereomer; selected data are shown below for the minor isomers (Table 4). ${ }^{1} \mathrm{H}$ NMR $\left(500 \mathrm{MHz}, \mathrm{CDCl}_{3}\right) \delta 7.58(\mathrm{~d}, J=7.5$ $\mathrm{Hz}, 2 \mathrm{H}), 7.52(\mathrm{~d}, J=8.0 \mathrm{~Hz}, 2 \mathrm{H}), 7.42(\mathrm{t}, J=8.0 \mathrm{~Hz}, 2 \mathrm{H}), 7.33-7.30(\mathrm{~m}, 3 \mathrm{H}), 4.16(\mathrm{t}, J=7.5 \mathrm{~Hz}, 1$ H), 1.91-1.85 (m, $1 \mathrm{H}), 1.79-1.72(\mathrm{~m}, 1 \mathrm{H}), 1.68-1.54$ (m, $2 \mathrm{H}), 1.30$ (s, $3 \mathrm{H}), 1.21$ (s, $6 \mathrm{H}) ;{ }^{2} \mathrm{H}$ NMR $\left(83 \mathrm{MHz}, \mathrm{CHCl}_{3}\right) \delta 2.94(\mathrm{~s}, 1 \mathrm{D}) ;{ }^{13} \mathrm{C} \mathrm{NMR}\left(125 \mathrm{MHz}, \mathrm{CDCl}_{3}\right) \delta 143.6,141.1,138.8,128.6,128.5$, $126.9,126.8,126.7,82.4,80.5,43.3\left(\mathrm{t}, J_{\mathrm{CD}}=19.3 \mathrm{~Hz}\right), 38.4,28.7,28.5,27.9,16.3$; IR (film) 1364 $\mathrm{cm}^{-1}$. MS (ESI) $m / z 304.1788$ (304.1787 calcd for $\mathrm{C}_{20} \mathrm{H}_{23} \mathrm{DNaO}, \mathrm{M}+\mathrm{Na}^{+}$).

Table 4. Selected NMR Data for the mixture of 32,33 , and 30

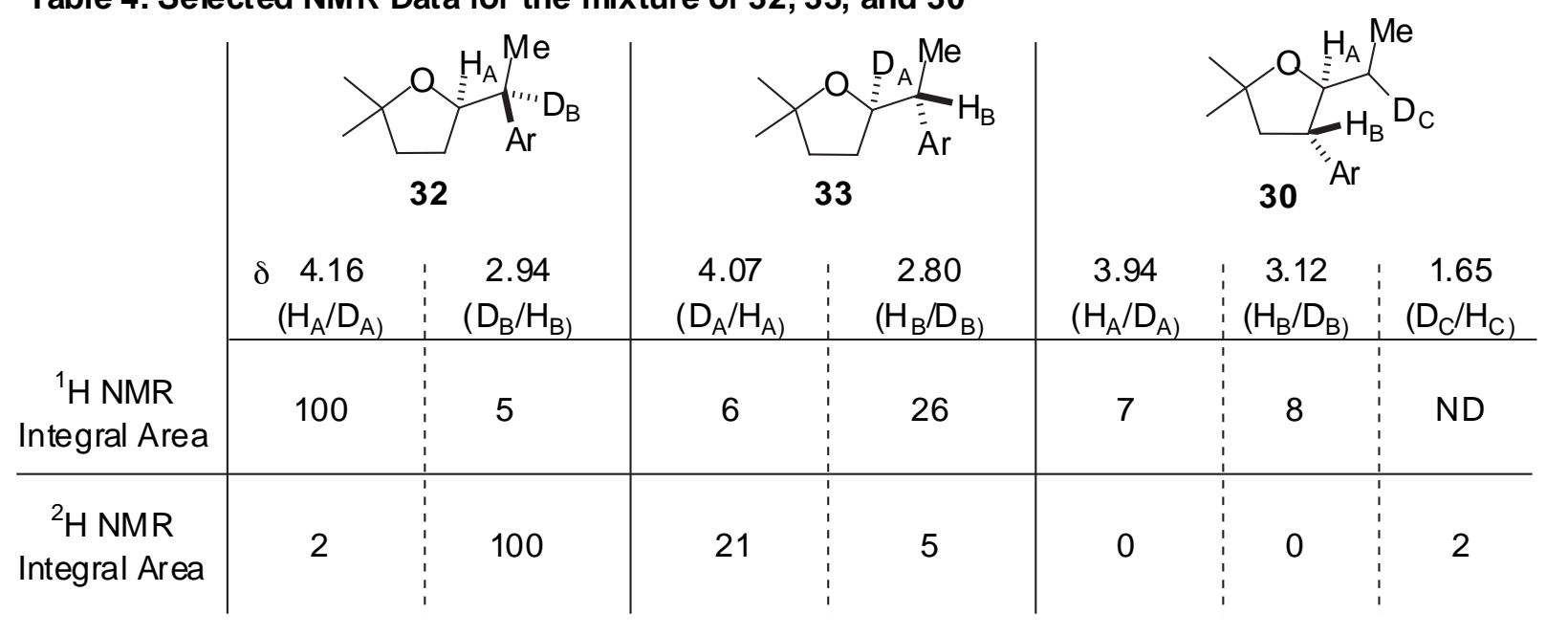

(E)-7,7,7-Trideuterio-2-methylhept-5-en-2-ol (34). A flame-dried flask was cooled under a stream of argon and charged with magnesium turnings $(3.60 \mathrm{~g}, 150 \mathrm{mmol})$. The flask was purged with argon, diethyl ether $(25 \mathrm{~mL})$ was added, and the resulting suspension was cooled to $0{ }^{\circ} \mathrm{C}$. 
Trideuteriomethyl iodide $(7.25 \mathrm{~g}, 50 \mathrm{mmol})$ was added dropwise and the resulting mixture was warmed to rt and stirred for $2 \mathrm{~h}$. The resulting solution was transferred to a dry, argon filled flask via cannula and additional diethyl ether $(20 \mathrm{~mL})$ was added by syringe. A solution of acrolein $(2.10 \mathrm{~g}, 37.6$ mmol $)$ in diethyl ether $(10 \mathrm{~mL})$ was cooled to $0{ }^{\circ} \mathrm{C}$ and added dropwise to the Grignard solution. The resulting mixture was stirred at $\mathrm{rt}$ for $8 \mathrm{~h}$, and then saturated aqueous $\mathrm{NH}_{4} \mathrm{Cl}(30 \mathrm{~mL})$ was added dropwise. The reaction mixture was diluted with diethyl ether $(40 \mathrm{~mL})$ and the aqueous layer was removed and extracted with diethyl ether $(2 \times 20 \mathrm{~mL})$. The organic layers were combined and dried over anhydrous $\mathrm{Na}_{2} \mathrm{SO}_{4}$, decanted, and concentrated by fractional distillation to afford $607 \mathrm{mg}(21 \%)$ of 1,1,1-trideuteriobut-3-en-2-ol. ${ }^{1} \mathrm{H}$ NMR (400 MHz, $\left.\mathrm{CDCl}_{3}\right) \delta 5.91$ (ddd, $J=6.4,10.4,17.0 \mathrm{~Hz}, 1 \mathrm{H}$ ), $5.20(\mathrm{~d}, J=17.2 \mathrm{~Hz}, 1 \mathrm{H}), 5.06(\mathrm{~d}, J=10.4 \mathrm{~Hz}, 1 \mathrm{H}), 4.28(\mathrm{~d}, J=5.6 \mathrm{~Hz}, 1 \mathrm{H}), 1.62(\mathrm{~s}, 1 \mathrm{H})$.

1,1,1-Trideuteriobut-3-en-2-ol $(607 \mathrm{mg}, 8.1 \mathrm{mmol})$ was treated with triethylorthoacetate according to general procedure 2 to afford $600 \mathrm{mg}(51 \%)$ of $(E)-6,6,6$-trideuteriohex-4-enoic acid ethyl ester as a pale yellow liquid. ${ }^{1} \mathrm{H}$ NMR $\left(500 \mathrm{MHz}, \mathrm{CDCl}_{3}\right) \delta 5.45-5.39(\mathrm{~m}, 2 \mathrm{H}), 4.12(\mathrm{q}, J=7.0$ Hz, 2 H), 2.36-2.32 (m, 2 H), 2.31-2.27 (m, 2 H), 1.24 (t, J=7.0 Hz, 3 H).

(E)-6,6,6-trideuteriohex-4-enoic acid ethyl ester (600 mg, $4.1 \mathrm{mmol})$ was treated with $\operatorname{MeMgBr}(6.8 \mathrm{~mL}, 20.5 \mathrm{mmol}, 3.0 \mathrm{M}$ solution in diethyl ether) according to general procedure 1 to afford $325 \mathrm{mg}(61 \%)$ of the title compound as a clear oil. ${ }^{1} \mathrm{H}$ NMR $\left(500 \mathrm{MHz}, \mathrm{CDCl}_{3}\right) \delta 5.45-5.44(\mathrm{~m}$, $2 \mathrm{H}), 2.09-2.04(\mathrm{~m}, 2 \mathrm{H}), 1.54-1.51(\mathrm{~m}, 2 \mathrm{H}), 1.30(\mathrm{~s}, 1 \mathrm{H}), 1.21(\mathrm{~s}, 6 \mathrm{H}) ;{ }^{2} \mathrm{H}$ NMR $\left(83 \mathrm{MHz}, \mathrm{CHCl}_{3}\right)$ $\delta 1.58$ (s, 3D); ${ }^{13} \mathrm{C}$ NMR (100 MHz, $\left.\mathrm{CDCl}_{3}\right) \delta 131.4,124.7,70.8,43.3,29.1,27.5,17.2$ (m); IR (film) $3369 \mathrm{~cm}^{-1}$; MS (EI) $m / z, 113.1285$ (113.1281 calcd for $\mathrm{C}_{8} \mathrm{H}_{13} \mathrm{D}_{3} \mathrm{O}-\mathrm{H}_{2} \mathrm{O}$ ).

\section{Palladium-Catalyzed Reaction of $(E)$-7,7,7-Trideuterio-2-methylhept-5-en-2-ol with 4-}

bromobiphenyl (eq 5). Reaction of $64.5 \mathrm{mg}(0.5 \mathrm{mmol})$ of (E)-7,7,7-trideuterio-2-methylhept-5-en-2- 
ol with 4-bromobiphenyl (233 mg, $1.0 \mathrm{mmol})$ according to the general procedure afforded $100 \mathrm{mg}$ (70 $\%)$ of $( \pm)-(1 ' S, 5 R)-5$-[1'-(4-Phenyl)phenylethyl]-2,2-dimethyltetrahydrofuran-D-3 The material was isolated as a ca. 71:23:6 mixture of $\mathbf{3 5 : 3 6 : 3 7}$ as judged by ${ }^{1} \mathrm{H}$ NMR analysis; all isomers contained three deuterium atoms as judged by MS analysis. All three isomers were trideuterated at the C1' methyl group. Complete data are given for the major diastereomer 35. ${ }^{1} \mathrm{H} \mathrm{NMR}\left(500 \mathrm{MHz}, \mathrm{CDCl}_{3}\right) \delta$ $7.65(\mathrm{~d}, J=7.0 \mathrm{~Hz}, 2 \mathrm{H}), 7.59(\mathrm{~d}, J=8.0 \mathrm{~Hz}, 2 \mathrm{H}), 7.48(\mathrm{t}, J=8.0 \mathrm{~Hz}, 2 \mathrm{H}), 7.40-7.37(\mathrm{~m}, 3 \mathrm{H}), 4.17$ $(\mathrm{q}, J=6.0 \mathrm{~Hz}, 1 \mathrm{H}), 2.92(\mathrm{~d}, J=5.5 \mathrm{~Hz}, 1 \mathrm{H}), 1.90-1.84(\mathrm{~m}, 1 \mathrm{H}), 1.78-1.70(\mathrm{~m}, 1 \mathrm{H}), 1.67-1.51(\mathrm{~m}$, $2 \mathrm{H}), 1.21(\mathrm{~s}, 6 \mathrm{H}) ;{ }^{2} \mathrm{H}$ NMR $\left(83 \mathrm{MHz}, \mathrm{CHCl}_{3}\right) \delta 1.30(\mathrm{~s}, 3 \mathrm{D}){ }^{13} \mathrm{C}$ NMR $\left(100 \mathrm{MHz}, \mathrm{CDCl}_{3}\right) \delta 143.2$, $141.1,138.8,128.6,128.5,126.9,126.8,126.7,82.5,80.5,43.5,38.4,28.7,28.5,27.9,16.3$ (m); IR (film) $1364 \mathrm{~cm}^{-1}$. MS (ESI) $\mathrm{m} / z 306.1913$ (306.1910 calcd for $\mathrm{C}_{20} \mathrm{H}_{21} \mathrm{D}_{3} \mathrm{NaO}, \mathrm{M}+\mathrm{Na}^{+}$).

\section{References}

${ }^{1}$ Wolfe, J. P.; Rossi, M. A. J. Am. Chem. Soc. 2004, 126, 1620-1621.

${ }^{2}$ Johnson, W. S.; Werthemann, L.; Bartlett, W. R.; Brocksom, T. J.; Li, T.; Faulkner, D. J.; Petersen, M. R. J. Am. Chem. Soc. 1970, 92, 741-743.

${ }^{3}$ Baskaran, S.; Islam, I.; Chandrasekaran, S. J. Chem. Res. 1992, 2213-2246.

${ }^{4}$ Bestmann, H. J.; Koschatzky, K. H.; Schaetzke, W.; Suess, J.; Vostrowsky, O. Liebigs Ann. Chem. 1981, 9, 1705-1720.

${ }^{5}$ Huber, R. S.; Jones, G. B. J. Org. Chem. 1992, 57, 5778-5780.

${ }^{6}$ Bradbury, B. J.; Sindelar, R. D. J. Heterocyclic Chem. 1989, 26, 1827-1833.

${ }^{7}$ Adah, S.H.; Nair, V. Tetrahedron 1997, 53, 6747-6754.

${ }^{8}$ Tamaru, Y.; Ochiai, H.; Nakamura, T.; Yoshida, Z. Tetrahedron Lett. 1986, 27, 955-958.

${ }^{9}$ Loh, T. -P.; Hu, Q. -Y.; Ma, L. -T. J. Am. Chem. Soc. 2001, 123, 2450-2451.

${ }^{10}$ Padwa, A.; Brodney, M. A.; Lynch, S. M. Org. Synth. 2000, 78, $202-211$.

${ }^{11}$ Palucki, M.; Buchwald, S. L. J. Am. Chem. Soc. 1997, 119, 11108-11109.

${ }^{12}$ Barentson, H. M.; Talman, E. G.; Piet, D. P.; Cornelisse, J. Tetrahedron 1995, 51, 7469-7494. 